\title{
Research and development on ticketing system based on SQL database
}

\author{
Yuntao Duan \\ Software College \\ Nanyang Institute of Teclanology \\ Nanyang, China
}

\author{
Siping $\mathrm{Hu}$ \\ School of Logistics Engineering \\ Wuhan University of Technology \\ Wuhan, China \\ zjlg0001@126.com
}

\begin{abstract}
Currently, all of the transport sectors such as the aviation, rail, highway, marine and other areas' automated ticketing system have been basically able to quickly meet the needs of people-smart, saving manpower and material resources. However, small businesses and individuals need to further expand its function; they need secondary development on this system to meet their financial management, personnel management, customer information management. It is because there is a strong demand for a large number of small travel agencies and individual outlets that the system has good prospects. The current version is $\mathbf{5 . 0}$, through several versions upgrade and bug fixes, the current version is very stable and the reconciliation has basically no error.
\end{abstract}

Keywords-SQL; ticketing system; information management; database

\section{BACKGROUND AND SIGNIFICANCE}

With the deepening of reform and opening up, and the continuous improvement of people's living standards, enormous change has undergone in the way people travel. In the past, people did not even think of travelling by plane, at present that means of transportation has already moved into the homes of ordinary people.

Statistics show that in new China first established in 1950 , the CAAC has only 7 domestic routes, navigate 8 cities in China. China civil aviation transportation turnover, passenger traffic, cargo and mail transport volume were separately 1.57 million ton-km, 10,000 people and 767 tons.

In 2008, China's civil aviation industry-wide total transportation turnover, passenger traffic, cargo and mail traffic volume reached 37.6 billion ton kilometers, 192 million people and 4.07 million tons respectively.

By 2012, despite of the deep impact of the international financial crisis and the world economic slump, China's civil aviation transportation index maintained fast and steady growth. In 2012 Civil Aviation reached the industry-wide total turnover of 61.1 billion ton-km, passenger traffic 320 million people, which respectively grew 5.8\% and 9.2\% over the previous year.

Currently the main way people buy tickets are: 1 、 book tickets online 2、 phone orders 3、 buy in the airport hall 3、 go to the ticket shop; The first three are of small proportion; book on ticket shop is the main channel. Many ticket outlets emergence such as travel agency. Usually there is numerous ticket outlets places, customers are generally acquaintances, new customers are mostly introduced by old customers, which is much more safe and reliable. At the same time, its telephone booking and home delivery service are very attentive. Thus, attract a wide customer base.

However, to travel agents and other ticket outlets, the major airlines ticket sales is a large independent ticketing system, they just use one simple function of the complex system-booking tickets. Considering from the security perspective, airline ticketing system will not provide an interface to an independent small ticket sales point to realize their personality development. Such proxy like travel agency, hope that in addition to the ticket booking function, the system can also record the customer information to achieve a certain degree of data mining, further implementation of financial statistics, financial closure and some other functions, even achieve the members' business evaluation. In the past, these are done by hand, the statistical process is very cumbersome which is a huge workload.

In the ticket information, often there are customers return or change the ticket, which requires the clerk to find the corresponding information and record the modification making the workload very tedious. While some salesmen resign or leave, other jobs staff should take over the recording task which is a very troublesome thing as well.

Many customers sometimes find themselves did not take them with enough money when booking, or through telephone booking; some customers are staff of enterprises and institutions, they tend to be in arrears or pay partial payments, so there will be arrears information; also some customers do not pay off the debts at one time, so there will be multiple payments information. Thus, recording the arrears and summary information on debts of each clerk becomes very cumbersome. The repeated deletion and multiple repayment dates seen from the figure can prove the above fact.

Financial staff has to do the reconciliation information table every day, they need to check the statistical information and the actual receipt of money in order to see whether the accounts balance, or they need to check again. Sometimes they need to re-do several times.

Therefore, for such as travel agency ticketing point, developing a kind of ticketing system meeting the above needs is of important meaning. 


\section{RELATED CONCEPTS AND TECHNOLOGIES}

\section{A. NET Framework}

\section{1) NET Framework Introduction}

Microsoft.NET developer brings out a new development framework-NET Framework. Before NET Framework released, program interfaces is Win32 AP and COM object, ASP pages interface is the object provided by asp.dll. However, after NET Framework release, both program and Web pages interface are class library provided by NET Framework.

- NET development platform allows developers to create applications that run on IIS Web server easier. At the same time, it can create a stable, reliable and secure Windows desktop application more quickly. NET Framework contains all the layers in the software development on operating system. It is a high degree integration of display technology, component technology and data technology on Microsoft or other platforms.

\section{2) Winform introduction}

WinForm is a title for Windows Form on Net development platform. NET Framework is the basement of NET platform, which combines the Common Language Runtime and libraries, and link library contains Windows Forms, Active Data Object and ASP.NET [14] Figure 2.3 shows the relationship between WinForm and. NET Framework.

Windows Forms is a new application design technology based on the Windows platform. The essence of the technique is also based on NET Framewok, the so-called Rich windows client Library. Using this new technology can fully utilized underlying support of NET platform such as NET Class Library object-oriented features and a variety of services provided by the CLR, etc.to develop Windowsbased applications. Windows Forms can also be used to develop local interface of a distributed system with multilayer structure.

WinForm has the following characteristics:

1. Simple and powerful: Windows Forms can be used to design forms and visual controls to create rich Windowsbased applications.

2. The new data provide Program Management: Data Management provides data controls which can connect OLEDB and ODBC data source easier, including Microsoft SQL Server, Microsoft Access, Jet, DB2 and Oracle, etc..

3. Security: Windows Forms take full advantage of the security features of the common language runtime. This means that everything can be achieved through Windows Forms, including incredible control from the browser and completely trusted user-installed applications on the hard disk.

4. Flexible controls: Windows Forms provides a rich set of controls, and developers can define their own distinctive new controls.
5. Wizard: to provide users with distribution guidance to create forms, data processing, packaging and disposition etc.

3) SQL Server 2008 Introduction

SQL Server 2008 is a client / server architecture relational database management system (RDBMS) developed by Microsoft, which uses Transact-SQL (after Microsoft expanded database query language - SQL) to transfer request and response [16] between SQL Server client and server.

1. Client/server architecture

The client program is responsible for the implementation of business logic and the display of user interface, it can run on one or more clients, it can also run on SQL Server 2008 server.

SQL Server 2008 database server is responsible for the management and allocation of server resources available across multiple user requests, such as memory, network bandwidth and disk operations, etc..

The software is powerful, easy to use, has a large user community, it is the best choice for back-end database server.

2. Relational database management system (RDBMS)

RDBMS is responsible for constructing the relational model database, specifically including maintaining relationships between data and information; under the premise that it does not violate the rules defining the relationship between data to ensure data's correct access; in the case of system failure, it will restore all consistent data to a known point, etc. [18].

\section{Transact-SQL}

SQL Server 2008 using Transact-SQL as its database query and programming language, using Transact-SQL language, you can access the data, query, update, and manage relational database system. Transact-SQL supports the latest ANSI SQL international standards, and increases a number of extensions to provide additional functions.

SQL Server 2008 provides two of the most important tools for database development and management, namely Enterprise Manager and Query Analyzer.

Enterprise Manager is the most important tool that can almost completed the development and management of all SQL Server 2008 database, through Enterprise Manager, developers can improve the efficiency of database development and administration.

Query Analyzer is a database developer's favorite tool, the tool database developers can directly use the SQL language to interact with SQL Server 2008, you can create views, stored procedures triggers and other database objects through SQL statements, you can debug SQL statement to the correct SQL statements embedded in an application. 


\section{SYSTEM REQUIREMENTS ANALYSIS AND DESIGN}

\section{A. System design goal}

Ticket purchasing system's main function is to provide ticket purchasing, billing and other related content for staff and managers in purchasing points, to achieve the best purchasing results.

1. Its functions are described below:

(1) The management and staff's registration and login on the system.

(2) Passengers, flight, billing and other related information's input and store records.

(3) The administrator of employee information, billing information, customer information, order information and refund management.

(4) Staff responsible for their own billing information, customer information, order information, and refund management.

2. Conditions and restrictions

(1) Constraints of manpower, money, time: Target ticket purchasing system's implementation is to give companies more profits and more convenient. The development process should take into account the constraints of manpower, resources and time. Focused on improving the system statistics timeliness, accuracy and convenience, bring better benefits to the ticket purchasing point.

(2) Technology development law: the rapidly changing development of computer technology and products will bring more means on information processing, enriching expression of information, which replace the tedious paper documents operation processes.

\section{B. Performance requirements}

\section{Data Accuracy}

When extracting data from the database, requiring accurate positioning data; when entering data into the database, requires data input validation accuracy; query return information is derived from the database.

\section{Time Characteristics}

Update processing, data conversion and transmission, and run-time operation will have different reactions affected by individual's specific operation on equipment; and with system constantly updated and version upgrade (hardware conditions allowed) the situation will be improved.

\section{Adaptability}

When demand changes, the ticket purchasing management application software's operation, basic operating environment does not change, changes only exist in the corresponding database record within the file, or you can change the filter.

\section{Operating environment requirements}

1. Device Server

Hardware: CPU133M, 64M or more RAM, 300M hard disk space; VGA monitor or higher
Client computer

Hardware: minimum CUP133M,16MB RAM; least 8MB of

RAM memory; VGA monitor or higher

2. Software Support

Client computer

Software: Windows 95 or higher or; IE 4.0 above

The software runs on a Windows system, database management system is SQL Server, developed by C \# and other languages

3. Hardware interface

This product does not require specific hardware engineering or hardware interface support

\section{Software Interface}

The product's software interface is the composition of programming operating system, SQL database; air tickets management applications' dictionaries and data structures.

\section{Controls}

Operational control will be in strict accordance with the relationship between the module function to achieve. In the center of each transaction modules, it required for proper judgment of operation control, choose the correct operation control path. In terms of network transmission, after the client sending data, the server will wait for confirmation of receipt of the signal, upon receipt, waiting for the server to send response data again, and then confirm the data. After receiving the data server sends a confirmation signal, then the data processing, get access to the database, at last return information back to the client, and wait for confirmation.

\section{System Development and depend environment}

\section{1) Development Environment}

The system is developed under the microsoft's fromwork environment. The system is developed under FormWork4.0 environment version 1, support those whose interfaces requirement complying with microsoft's fromwork 3.0 or the above version. Development language used c \# language. Using sql server2008 database to develop, database sql server 2008 or the above version are supported.

\section{2) Dependencies}

This program's executable file is AirTicket.exe. It is developed under NET FormWork framework, VS2010 environment, depend on database SQL Server2008. All of its interfaces libraries are from NET FormWork library. The program is divided into three tiers, the interface is mainly developed by vs 2010 winform, behind language mainly written in c\# code, and database depends on SQL SERVER2008.

\section{Database Design}

\section{A. Overall system architecture}

The system uses a classic three-tier model, the system is divided into three layers, from bottom to top are: data access layer, business logic layer and the presentation layer, the following will detail the overall architecture of the 
information management system, the system allows us to deeply understand the overall architecture.

Data Access Layer: its main function is responsible for accessing the database. Simply put, is to realize the data table Select, Insert, Update, Delete operations.

Business logic layer: the core of the whole system, which is related to this system's business. In the ticketing system, related design in is associated with the daily management process. Business logic layer send request through interface responds presentation layer, then complete the corresponding operations process and finish operating data through the interface data access layer.

Presentation Layer: UI part of the system is responsible for the interaction between the user and the system. In the presentation layer, the ideal state is not supposed to contain the business logic of the system, which means that the logic code only related to the elements of page.

Data sets in the system architecture belongs to entity class, entity class in fact corresponds to a table in the database, each entity class property mapping table corresponding field. The benefit of using the three-tier entity class is that when modified field names in database it can just modify the data layer code without affecting other layers or associated data interface.

\section{B. The main function module design}

\section{1) Staff Management Module}

In the ticket management system, staff management module can query the basic information for all employees; different staff has different operating authority so as to ensure that unauthorized users cannot use the system.

\section{2) Billing management module}

Billing management is used to manage the daily reconciliation of bills, including a bill deadline day total debt, total cash received that day, the total individual bank cards and electronic accounts, airline refunds, fees, etc.. This module is the core module of the system, is automatically generated by the system daily to facilitate financial reconciliation.

\section{3) Customer Management Module}

Customer information management module can complete basic customer information (including customer ID number, customer name, customer phone, the company address information, etc.). In this module information is automatically generated during the customers' leaving and booking process, mainly for flight delays, cancellations and ticket delivery, and situations like that.

\section{4) Order Management Module}

Order Management module is also a core module in the system, the module is mainly for order entry, achieve the management of orders. Orders including order number, ticket number, route, passenger information. Each order can include more than one ticket. Order information is source of information throughout the system.

5) Owe repayment management module
Since during the booking process, many customers book through telephone or cash is not enough, there is no payment or partial payment of money orders, so the main function of this module is used to manage customer arrears statistical information, the main purpose is to be reconciled and pay the repayments. This module contains information like customer ID, name, order number, amount owed, the repayment date, etc.

The module supports multiple batches customer repayment until the debt paid off. The arrears list also supports export to excel, easy to print and submit reference leadership decisions.

6) Change tickets and unsubscribe management module

In the actual process, due to various reasons, the customer needs or unsubscribe ticket, so the module handling for the unsubscribe ticket. Unsubscribe ticket orders should be directly removed, and reduce the number of the ticket order. Change tickets should check the corresponding orders, and modified order information.

\section{System database design}

According to the needs analysis, ticket purchasing system entity attributes of each module are as follows:

1 Staff Management Entity Attribute: Staff ID, employee name, employee gender, ID number, telephone employees, full address, date of entry, the state in the post, photos, permissions, and password

2 Bill management entity attribute: date, the staff numbers, the total amount owed, cash, PayPal, ICBC, Bank, money paid through airline refund total, more than the carrying amount of the balance, refund money, the total amount due, the actual ticket profits, fees

3 Customer management entities attributes: ID number, the customer calls, customer zip code, detailed address, company

4 Booking information management entity attributes: starting order number, ticket number, route, flights day, votes, comments, system number

5 Due repayment management entity attributes: ID number, amount owed, date arrears repayment amount, the repayment date, employee number, notes, order number, outstanding balance

6 Order Management Entity Attribute: order number, total bookings, ID number, staff number, date, notes

\section{Database queryoptimization}

SQL query is a descriptive language, Inferior quality SQL statements and SQL statements' execution speed can reach tens of times more. The main factor affecting the efficiency of SQL queries are: 1 whether built with valid appropriate available index; 2 whether to optimize the SARG search parameters.

Improved methods are:

1. Establish the necessary indexes. Improve query efficiency, first establish indexed by the appropriate fields. Appropriate index, the query optimizer can accord the 
definition of the index to improve search efficiency, avoid full table scan inefficiencies.

2. Do not use SELECT * FROM table statement, must clearly identified the field.

3. Use the inner limiting principle, spelling SQL statement; try to limit most of the SQL statement in the inner layer where to reduce the amount of processing data.

4. Should definitely avoid expression in order by clause.

5. If you need to read data from related tables, relationships table are generally below seven.

6. If there is not in (in) operating in the statement, you should consider use not exists (exists) instead, the best way is to use an outer join to achieve.

7. When dealing with a business process, you should make the time between the start and end of things intervals as short as possible, so that in principle, the database read operation is completed in front, behind that is the database writes operation so as to avoid cross.

8. Uses union instead of or, will mostly make the cases much faster.

9. Count (*) (non-primary key columns faster) compared with the count, count is the fastest.

10. If a stage can be used as scanning parameters SARG (Searchable Arguments), then the call can be optimized, and you can use the index to quickly obtain the required data.

11. Properly use SARG. SARG defined: the search for limiting an operation, since it usually refers to a particular match. Form is as follows: Column Name operator <constant or variable> or <constant or variable> operator column name; column names can appear in the operator's side, and constant or variable appears in the other side of the operator.

\begin{tabular}{|l|l|}
\hline Examples of non-SARG parameters & Examples of SARG parameters \\
\hline like '\%xyz\% & \\
\hline in("'x","y",'z") & \\
\hline substring(name,2,6)='Mar' & like 'Mar\% \\
\hline ABS(Num-100)<1 & Num $>80$ and Num<120 \\
\hline salary $* 12>=2000$ & salary $>=5000 / 16$ \\
\hline$x !=10$ & $\begin{array}{l}\text { Select } * \text { from } t \text { where } x>12 \\
\text { Union all } \\
\text { Select } * \text { from } t \text { where } x<8\end{array}$ \\
\hline
\end{tabular}

\begin{tabular}{|l|l|}
\hline $\begin{array}{l}\text { select num from } \mathrm{x} \text { where num } \\
\text { in(select num from } \mathrm{y})\end{array}$ & $\begin{array}{l}\text { select num from } \mathrm{x} \text { where } \\
\text { exists(select num from } \mathrm{y} \text { where } \\
\text { num=x.num) }\end{array}$ \\
\hline select id from $\mathrm{t}$ where num is null & select id from $\mathrm{t}$ where num $=0$ \\
\hline
\end{tabular}

\section{CONCLUSION}

In this paper, a ticketing systems appropriate for travel agencies, private enterprises and individuals is researched and de veloped, analyzes its main requirements to develop the system and carried out a detailed test, version is in continuous improvement and upgrade, the current version has been on the line and has been well used in the actual environment. Currently, all of the transport sectors such as the aviation, rail, highway, marine and other areas' automated ticketing system have been basically able to quickly meet the needs of people-smart, saving manpower and material resources. However, small businesses and individuals need to further expand its function; they need secondary development on this system to meet their financial management, personnel management, customer information management. It is because there is a strong demand for a large number of small travel agencies and individual outlets that the system has good prospects. The current version is 5.0, through several versions upgrade and bug fixes, the current version is very stable and the reconciliation has basically no error.

\section{REFERENCES}

[1] http://news.carnoc.com/list/239/239849.ht ml

[2] Girigi Deogratias. Design and Implementation of A Multi-Tier Application Architecture: Hotel Booking System, Central South University, 2003.

[3] Foster L. Service-Oriented Science. Science, 2005, 308(5732):814817.

[4] Fernandez-Martinez J.L., Garcia-Gonzalo E. Stochastic stability analysis of the linear continuous and discrete PSO models. Evolutionary computation, 2011, 15(3):405-423

[5] Elisabeth Freeman. Head First Design Patterns, O'Reilly Media, 2004.M. Young, The Technical Writer's Handbook. Mill Valley, CA: University Science, 1989.

[6] James W.Cooper.C\# Design Patterns, Addison-Wesley Educational Publishers Inc,2002 